\title{
Derecho a la investigación y nuevas formas de difusión del conocimiento científico: El “Open Access”
}

\section{The Right to Research and the New Ways of Disseminating Scientific Knowledge: "Open Access"}

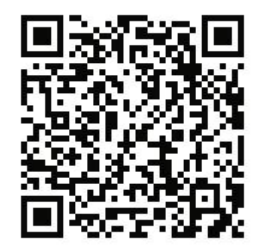

Jesús de Benito-Castanedo ${ }^{7}$

Santander, Cantabria, España

jdbcastanedo@hotmail.com

Recibido 2 de marzo de 2015 • Corregido 9 de julio de 2015 • Aceptado 31 de julio de 2015

Resumen. Se presenta un artículo que plasma una introducción al movimiento de acceso abierto (Open Access) y las principales características y herramientas que de él se derivan. Además, se conciencia sobre la importancia del acceso a la investigación y al conocimiento científico.

Palabras claves. Derecho a la investigación, difusión del conocimiento científico, acceso abierto.

Abstract. This article embodies an introduction to the "open access" movement and the main features and tools derived from it. In addition, it raises awareness about the importance of access to research and to scientific knowledge.

Keywords. Right to research, scientific knowledge dissemination, open access.

Nadie puede negar que el acceso a la investigación sea un derecho, así como poder difundir los resultados de trabajos científicos de manera sencilla, libre y gratuita.

El derecho a la investigación científica se reconoce en la constitución española, en su art. 44.2 que cita textualmente: "los poderes públicos promoverán la ciencia y la investigación científica y técnica en beneficio del interés general" (Constitución española, 1978, párr. 6).

Respecto a la difusión de la ciencia y derecho a la investigación, Chueca (2008), catedrático de derecho constitucional, sostiene que la investigación no necesariamente tiene que tener una finalidad concreta o una aplicación práctica para considerarla un derecho. La propia acción de investigar es el objeto de derecho.

' Diplomado en Educación Social por la Universidad del País Vasco. Máster Oficial en Investigación e Innovación en Contextos Educativos por la Universidad de Cantabria. 
doi: http://dx.doi.org/10.15359/ree.19-3.4

URL: http://www.una.ac.cr/educare

CORREO: educare@una.cr

También deja claro que es criterio de quien investiga el determinar el objeto de investigación, la elección del método que estime adecuado, la elección de los recursos que empleará y cualquier otro componente en su estudio; todo ello se encuentra amparado por la ley. $\mathrm{O}$, dicho de otro modo, "cada uno de estos componentes constituyen el objeto sobre el que se proyecta la protección constitucional" (Chueca, 2008, p. 10).

En lo relativo a la difusión de los hallazgos, expone que "el producto [del] trabajo de averiguación de la verdad es objetivable y por tanto comunicable" (Chueca, 2008, p. 9).

Pues bien, ya tenemos claro que el acceso a la investigación científica y la divulgación de sus correspondientes resultados son reconocidos derechos fundamentales.

Si preguntáramos a un investigador acerca, no solo del reconocido derecho a investigar, sino también de la necesidad de poder publicar sus hallazgos, sin coste, ni más barreras para acceder a ello que las que pueden suponer las tecnologías de la información y la comunicación, no habría duda en que la respuesta sería afirmativa.

Sin embargo, no todo ha sido tan fácil, ya que la persona investigadora se ha encontrado con diferentes restricciones para difundir sus estudios. Dejando de lado, aquellas barreas conocidas por cualquier investigador como puedan ser limitaciones de tipo metodológico o las impuestas por el controlador de acceso o sujeto que ha de darnos "luz verde" para comenzar a recabar datos, destacaremos tres.

En primer lugar nos encontramos con una barrera puramente material (¿Formato papel o mejor electrónico?). Anteriormente era necesario y no podía ser de otro modo, contar con un soporte en papel para poder difundir el conocimiento, formato que aún se encuentra vigente.

En segundo lugar hallamos una restricción económica, donde existen, incluso, editoriales que cobran altas tasas a los autores y autoras por publicar (¿Tengo que pagar por publicar?).

Y, por último, una traba jurídica (¿Tiene la revista todos los derechos sobre mi obra?). Aquí recordamos las restricciones de derechos que imponen las editoriales.

No obstante, en los últimos años, con la revolución tecnológica y la aparición de internet, surgen nuevas formas de difusión científica y, con ellas, el movimiento de Acceso Abierto, conocido también con el término en inglés "Open Access" (O.A.). Su finalidad es hacer realidad el anhelo de las personas investigadoras, de poder publicar sus estudios de manera sencilla, libre, gratuita, así como que su obra sea fácilmente accesible.

Por lo tanto, ello ha sido un tema tratado, además de por el discurso jurista que como hemos visto se postula claramente a favor de la investigación, por la comunidad académica en general que, en las últimas décadas, ha llevado a cabo importantes iniciativas con el fin de potenciar y regular el acceso abierto. De ello surgen, a su vez, nuevas herramientas y formas de divulgación digitales, tema que veremos posteriormente. 
doi: http://dx.doi.org/10.15359/ree.19-3.4

URL: http://www.una.ac.cr/educare

CORREO: educare@una.cr

Para tener una idea del concepto y de la dimensión del acceso abierto, citamos a Abadal (2012, p. 8), que expone lo siguiente:

[Es] un cambio de paradigma, una revolución que quiere modificar de pies a cabeza el sistema de comunicación de la ciencia... Es un movimiento que reclama la construcción de un dominio público para la ciencia y la cultura, que permita la difusión y reutilización del conocimiento y, por extensión, un rápido progreso científico y cultural.

Se destaca la iniciativa de Budapest del año 2002, que lanzó una campaña mundial para el acceso abierto (Open Access) y que dibuja las directrices de este novedoso modelo de publicación científica (Robinson-García, Delgado-López-Cózar y Torres-Salinas, 2011).

Esta iniciativa tiene como meta la creación de:

Un bien público sin precedentes" así como "acelerar la investigación, enriquecer la educación, compartir lo aprendido por los ricos con los pobres y lo aprendido por los pobres con los ricos, hacer que la literatura científica sea tan útil como pueda ser, y sentar las bases para unir a la humanidad en una conversación intelectual y búsqueda del conocimiento compartidas. (Budapest Open Access Initiative, 2002, párr. 3)

A estas alturas ya tenemos claro el concepto y la importancia que tiene no solo el derecho fundamental a la investigación, sino también la publicación en abierto. A continuación, veremos, de manera más detallada, las herramientas existentes para potenciarel acceso abierto en las publicaciones científicas, así como las modalidades de publicación que surgen del movimiento "Open Access".

Hemos de tener claras las dos formas en las que pueden ponerse a disposición pública los resultados científicos (Robinson-García et al., 2011).

En primer lugar, encontramos la llamada "Ruta Verde", cuyos ingresos son recibidos de los clientes, normalmente universidades y otras instituciones investigadoras y educativas, y no los propios autores o autoras.

Esta vía se caracteriza, también, por el establecimiento de caminos diferentes para acceder al manuscrito, y aboga por el "autoarchivo" como modo de difusión. Dicho de otro modo: es responsabilidad de la persona autora divulgar su trabajo en internet, además del propio artículo publicado en la revista ${ }^{3}$. Quien investiga está sujeto únicamente a las restricciones que la editorial pueda imponerle y recae sobre sí el asegurar el acceso al documento.

${ }^{2}$ El autoarchivo consiste en subir el trabajo a la web.

${ }^{3}$ Puede existir un "periodo de embargo", tiempo durante el cual es derecho exclusivo de la revista la explotación del manuscrito. 
doi: http://dx.doi.org/10.15359/ree.19-3.4

URL: http://www.una.ac.cr/educare

CORREO: educare@una.cr

Para facilitar el trabajo al autor o autora sobre las restricciones que cada revista impone y, por lo tanto, para saber qué versión de su trabajo debe poner a disposición pública, existen novedosas herramientas digitales. Se destaca el proyecto "Sherpa Romeo", que divide a las editoriales en cuatro grupos, dependiendo de las restricciones, y que el proyecto Romeo diferencia por colores.

Las editoriales clasificadas en color verde son las más permisivas, ya que dan vía libre al acceso al documento en cualquier forma (pre-print ${ }^{4}$ y post-print ${ }^{5}$ ).

Las catalogadas en color azul indican que será solamente la versión "post-print" la que podrá ser accesible al público, es decir, se permite el auto-archivo de la versión final del artículo, posterior a la revisión.

Las editoras amarillas permiten que sea difundida en la red, la versión "pre-print" de la obra.

Por último, encontramos aquellas revistas identificadas por el color blanco. Son las más restrictivas, ya que no comulgan con el acceso público y, por lo tanto, no ceden al autor o autora el derecho de publicar su obra en abierto, es decir, no permiten el autoarchivo (Robinson-García et al., 2011).

La herramienta "Sherpa Romeo" no se centra demasiado en el ámbito hispanohablante, para el cual existe el proyecto “Dulcinea”, que sigue criterios similares.

Por su parte, la vía dorada no se encuentra aún del todo perfilada y sugiere que las personas autoras paguen algún tipo de tasa para acceder al documento, como medio de manutención de la revista.

Respecto al número de revistas existentes en acceso abierto, con controles de calidad y que permiten al lector el acceso libre a texto completo de sus publicaciones, destacamos que en la actualidad rondan las 7.400 a nivel internacional: en España, 401. Por lo tanto, un 26\% del total de las revistas españolas se publican en abierto, según el Directory of Open Access Journals (conocido por sus siglas en inglés DOAJ) (Abadal, 2012).

Entre todas ellas, es importante diferenciar la validez científico-académica que tienen las publicaciones, pues es sabido que no todo lo que circula por internet tiene plena fiabilidad académica. Para ello, además de nuestro criterio personal, que como personal de investigación y conocedor de la temática podemos aplicar, es conveniente conocer los tres tipos de estándares de calidad (Abadal, 2012).

\footnotetext{
${ }^{4}$ Pre-Print: Versión del artículo previa a la revisión por pares.

${ }^{5}$ Post-Print: Versión del artículo, después de la revisión y con las correspondientes sugerencias incorporadas.
} 
doi: http://dx.doi.org/10.15359/ree.19-3.4

URL: http://www.una.ac.cr/educare

CORREO: educare@una.cr

En primer lugar, y es el que más garantías ofrece, encontramos el grupo conocido como "Élite". Con gran incidencia en la bibliografía anglosajona, podemos citar dos conocidas bases de datos: Scopus (19.000 títulos) y Web of Science (10.000), donde se encuentran indexadas las revistas que cumplen con los más altos estándares de calidad.

En segundo lugar, existen las revistas con revisión externa. A lo que algunos autores hacen mención con el término en inglés "peer review" (revisión por pares). En estas la validez científica de los artículos se determina siguiendo una valoración por equipos expertos en la respectiva temática, y ajenos a la revista. Existen unos 34.000 títulos en esta categoría.

Por último, destacamos las revistas sin revisión externa, en la que los escritos son revisados por el consejo editorial de la propia revista y poseen una naturaleza más divulgativa que científico-investigadora. Suponen un amplio abanico de 39.000.

Son reseñables, además, las bibliotecas digitales y portales de difusión, herramientas dignas de mención, cuando hablamos de divulgación científica, debido al amplio abanico de recursos a los que nos permiten acceder. Aquí destacan recursos como la biblioteca virtual Miguel de Cervantes y Dialnet, entre otros muchos. En esta última, además de visualizar artículos, podemos ver una clasificación científica de cada revista.

En el ámbito académico es posible encontrar diversos repositorios. En ellos se archivan documentos académicos y científicos como son los conocidos trabajos de fin de grado, trabajos fin de máster, tesis doctorales y otros manuscritos elaborados en el seno de cada universidad. Así mismo existe la plataforma "Recolecta” que aglutina a los diferentes repositorios académicos de universidades españolas, y el directorio OpenDoar, que contiene las obras de los repositorios existentes a escala mundial.

No debemos olvidar los buscadores de internet, como son los conocidos "Google académico", "Google Books" o"Microsoft academic search", que nos permiten acceder de manera sencilla a una inmensa cantidad de recursos electrónicos.

Tenemos en cuenta, por lo tanto, que la investigación y difusión científica, con reconocidos derechos fundamentales, han experimentado importantes cambios en las últimas décadas. Como personal investigador, académico y demás profesionales, deberíamos conocer, por lo menos básicamente, los nuevos recursos existentes para publicar nuestros resultados, así como apoyar la investigación científica y el acceso al conocimiento como derechos fundamentales. 
doi: http://dx.doi.org/10.15359/ree.19-3.4

URL: http://www.una.ac.cr/educare

CORREO: educare@una.cr

\section{Referencias}

Abadal, E. (2012). Acceso abierto a la ciencia. Barcelona : Editorial UOC. Recuperado de http://eprints. rclis.org/16863/1/2012-acceso-abierto-epi-uoc-vfinal-autor.pdf

Budapest Open Access Initiative. (2002). Diez años desde la Budapest Open Access Initiative: Hacia lo abierto por defecto (Trad. R. Melero y D. Babini). Recuperado de http://www. budapestopenaccessinitiative.org/boai-10-translations/spanish

Chueca, R. (Diciembre, 2008). El derecho fundamental a la investigación científica. REDUR, 6, 5-15. Recuperado de http://www.unirioja.es/dptos/dd/redur/numero6/chueca.pdf

Constitución Española (1978). La constitución española de 1978. Recuperado de http://www. congreso.es/consti/constitucion/indice/titulos/articulos.jsp?ini=39\&fin $=52 \&$ tipo $=2$

Robinson-García, N., Delgado-López-Cózar, E. y Torres-Salinas, D. (2011). Cómo comunicar y diseminar información científica en internet para obtener mayor visibilidad e impacto. Aula Abierta, 39(3), 41-50. Recuperado de http://ec3.ugr.es/publicaciones/aula abierta2011.pdf

\section{Cómo citar este artículo en APA:}

De Benito-Castanedo, J. (Setiembre-Diciembre, 2015). Derecho a la investigación y nuevas formas de difusión del conocimiento científico: El "Open Access". Revista Electrónica Educare, 19(3), 1-6. doi: http://dx.doi. org/10.15359/ree.19-3.4

Nota: Para citar este artículo en otros sistemas puede consultar el hipervínculo "Como citar el artículo" en la barra derecha de nuestro sitio web: http://www.revistas.una.ac.cr/index.php/EDUCARE/index 